\title{
The diagnostic value of three-dimensional CT angiography for patients with acute coronary artery disease
}

\author{
$\mathrm{LIBO} \mathrm{LI}^{1}, \mathrm{JING} \mathrm{HAO}^{2}$, SHI QU ${ }^{1}$ and YANCHENG FANG ${ }^{1}$ \\ ${ }^{1}$ Department of Radiology, The Affiliated Hospital of Changchun University of Chinese Medicine, Changchun, Jilin 130017; \\ ${ }^{2}$ Department of Pediatric Ultrasound, The First Hospital of Jilin University, Changchun, Jilin 130021, P.R. China
}

Received October 2, 2017; Accepted January 19, 2018

DOI: $10.3892 /$ etm.2018.6257

\begin{abstract}
Computed tomography angiography (CTA) is an efficient method for the diagnosis of heart disease. However, few contemporary studies have evaluated the prognostic value of three-dimensional (3D)-CTA for patients with acute coronary artery disease. The aim of the present study was to investigate the diagnostic value of 3D-CTA for patients with acute coronary artery disease. A total of 136 patients with suspected acute coronary artery disease were recruited and received conventional coronary angiography (CCA) and 3D-CTA. 3D-CTA was used to assess calcified plaques in the coronary arteries (CCTA), the ratio of calcified plaque volume to vessel circumference (RVTC) and diagnostic accuracy. The results revealed that 3D-CTA was a more effective diagnostic method for identifying calcified plaques in patients with acute coronary artery disease compared with CCA. 3D-CTA demonstrated a significantly better area under curve, sensitivity, specificity, positive predictive value and negative predictive value compared with $\mathrm{CCA}(\mathrm{P}<0.01)$. In the present study, 3D-CTA was used to successfully diagnose 86 patients with acute coronary artery disease, 34 with myocardial infarction and 16 with stable angina. 3D-CTA images clearly showed global noise levels and target-to-background ratios determined by manually delineated coronary plaque lesions compared with CCA. Furthermore, 3D-CTA was significantly better for discriminating ischemia compared with CCA $(\mathrm{P}<0.01)$. In conclusion, the results of the present study suggest that 3D-CTA provides superior diagnostic performance compared with CCA alone in patients with acute coronary artery disease.
\end{abstract}

\section{Introduction}

Coronary artery disease (CAD) is one of the most common complicated cardiovascular diseases and may be caused by

Correspondence to: Professor Yancheng Fang, Department of Radiology, The Affiliated Hospital of Changchun University of Chinese Medicine, 1478 Gongnong Road, Chaoyang, Changchun, Jilin 130017, P.R. China

E-mail: fangyanchengprof@163.com

Key words: 3D-computed tomography angiography, acute coronary artery disease, conventional coronary angiography atherosclerosis, vascular cavity stenosis and/or occlusion (1-3). It has previously been reported that acute CAD may contribute to the pathogenesis of occlusive thrombosis (4-6). Notably, multifactorial microvascular dysfunction in patients with acute CAD may result in blunted responses to adenosine and false-negative readings for fractional flow reserve (7). It is therefore important to develop more accurate diagnostic methods to confirm whether patients have CAD. In recent years, a number of diagnostic methods have been developed for coronary atherosclerotic heart diseases (8-10). Notably, cardiac computed tomography (CT) was reported to be more efficient compared with electrocardiogram and intravascular ultrasound in the diagnosis of coronary atherosclerotic heart diseases (11).

Currently, three-dimensional CT angiography (3D-CTA) is widely used to diagnose human tumors, heart diseases and other pathologic changes (12-15). It has been reported that 3D-CTA is an effective technique for imaging microvascular anatomy (16). 3D-CTA is able to identify vascular injuries to increase the diagnostic accuracy and improve treatment (17). Furthermore, 3D-CTA is essential for preoperative planning to decide on the best surgical approach to treat arteriovenous malformations (18). 3D-CTA diagnosis has been used as an initial screening tool in lieu of digital subtraction angiography to rule out vascular diseases in idiopathic spontaneous subarachnoid hemorrhage (19). Furthermore, a randomized controlled trial demonstrated that 3D-CTA is advantageous compared with conventional coronary angiography (CCA) when performing laparoscopic colorectal resection for patient's mesenteric vascular anatomy (20). However, the efficacy of 3D-CTA as a diagnostic tool for CAD remains to be elucidated.

The aim of the present study was to investigate the diagnostic efficacy of 3D-CTA and coronary CTA (CCTA), as well as the accuracy of CCTA in measuring calcifying plaques and the ratio of calcified plaque volume to vessel circumference (RVTC) in 136 patients with suspicious with acute CAD. The results demonstrated that $3 \mathrm{D}-\mathrm{CTA}$ has a superior diagnostic performance compared with CCA in patients with acute CAD.

\section{Materials and methods}

Study population. A total of 136 patients with suspected acute CAD were recruited from the Affiliated Hospital of Changchun University of Chinese Medicine (Changchun, China) between February 2014 and May 2015 and all patients underwent 
CCA and 3D-CTA (Discovery CT750 HD; GE Healthcare Life Sciences, Little Chalfont, UK) between May 2013 and October 2016. The exclusion criteria included renal insufficiency (serum creatinine level, $>1.5 \mathrm{mg} / \mathrm{dl}$ ), diabetes mellitus and a history of allergic reaction to contrast medium. The Ethical Committee of the Affiliated Hospital of Changchun University of Chinese Medicine approved this prospective study. Written informed consent was obtained from all the patients.

$C C A$. All patients were diagnosed using CCA. Calcified plaques were analyzed using quantitative CAA (AXIOM Artis dB; Siemens AG, Munich, Germany) (21). CCA was performed in all evaluable coronary segments to identify coronary stenosis and plaques by an experienced cardiologist, who was blinded to the conditions of the present study. The severity of stenosis was defined when lesions of the coronary artery were $\geq 50 \%$ in diameter.

3D-CTA analysis. 3D-CTA was performed to evaluate the lesions as previously described (22). All images were acquired using an MDCT scanner (Philips Healthcare, Amsterdam, The Netherlands) with 16 detectors and a tiltable gantry. The parameters negative predictive value (NPV), positive predictive value (PPV), CCTA and RVTC were recorded and analyzed using a dedicated cardiac workstation (TeraRecon $\mathrm{GmbH}$, Frankfurt, Germany). The lesions were analyzed on an OsiriX workstation (OsiriX Imaging Software 2.0; Pixmeo SARL, Bernex, Switzerland) and target-to-background ratio (TBR) was defined as the ratio of maximum activity values in the manually defined lesion to the mean background value. The plaque was confirmed by obvious presence of plaque or napkin ring signs and spotty calcium.

Statistical analysis. All statistical analyses were performed using SPSS 19.0 (IBM Corp., Armonk, NY,USA). Comparisons between groups were made using one-way analysis of variance for continuous variables followed by Tukey's post hoc test. Categorical variables were analyzed using Fisher's exact test and the sensitivity, specificity, NPV and PPV values were analyzed using exact binomial confidence intervals. For the CCTA assessment, the test was considered positive if $\geq 50 \%$ stenosis or plaque was identified. RVTC was analyzed using the receiver operating characteristics curve and the area under curve (AUC) was compared between groups using the DeLong algorithm. $\mathrm{P}<0.05$ was considered to indicate a statistically significant difference.

\section{Results}

Patient characteristics. A total of 136 patients with suspected acute CAD were recruited and diagnosed using 3D-CTA and CCA. Patients' baseline characteristics are summarized in Table I. No significant differences were observed in baseline demographics or risk factors for male and female patients. All patients underwent both 3D-CTA and CCA diagnosis.

Analysis of the calcified plaque and RVTC. The calcified plaques and RVTC were investigated in the present study. The results revealed that $3 \mathrm{D}-\mathrm{CTA}$ was able to identify calcified
Table I. Baseline patient characteristics.

\begin{tabular}{lcc}
\hline Characteristic & $\begin{array}{c}\text { Male } \\
(\mathrm{n}=80)\end{array}$ & $\begin{array}{c}\text { Female } \\
(\mathrm{n}=56)\end{array}$ \\
\hline $\begin{array}{l}\text { Mean age (years) } \\
\text { Cardiovascular risk factors (\%) }\end{array}$ & $53.4 \pm 13.3$ & $56.0 \pm 9.4$ \\
$\quad \begin{array}{l}\text { Hypertension } \\
\text { Dyslipidemia }\end{array}$ & $13(16.25)$ & $5(8.93)$ \\
Former or current smoker, n (\%) & $22(27.5)$ & $3(5.36)$ \\
Family history of premature coronary & $14(17.5)$ & $7(12.5)$ \\
artery disease, n (\%) & & $6(10.71)$ \\
Acute coronary syndrome, n (\%) & & \\
$\quad$ Myocardial infarction & $10(12.5)$ & $5(8.93)$ \\
Unstable angina pectoris & $8(10.0)$ & $5(8.93)$ \\
\hline
\end{tabular}

Table II. Diagnostic accuracy of 3D-CTA and CCA for patients with coronary artery disease.

\begin{tabular}{lccc}
\hline Variable & CCA & 3D-CTA & P-value \\
\hline Area under curve & 78.8 & 97.2 & 0.0385 \\
Positive predictive value (\%) & 71.2 & 94.3 & 0.0263 \\
Negative predictive value (\%) & 70.8 & 40.3 & 0.00170 \\
Specificity (\%) & 82.3 & 94.8 & 0.0478
\end{tabular}

3D-CTA, three dimensional computed tomography angiography; conventional coronary angiography.

plaques more clearly in patients with acute CAD compared with CCA (Fig. 1A and B). The calculated RVTC was higher using 3D-CTA compared with CCA for types III and IV $(\mathrm{P}<0.05$; Fig. 1C). These results suggest that 3D-CTA is an effective method for identifying calcified plaques and RVTC in patients with acute CAD.

$3 D$-CTA for identifying risk events in patients with acute CAD. The efficacy of 3D-CTA for identifying risk events (TBR and ischemia) was evaluated in patients with acute CAD. The results indicate that 3D-CTA diagnosis significantly improved the AUC, specificity, PPV and NPV compared with CCA diagnosis $(\mathrm{P}<0.05$; Table II). Global noise levels and TBR were significantly decreased using 3D-CTA compared with CCA ( $\mathrm{P}<0.05$; Fig. $2 \mathrm{~A}$ and $\mathrm{B})$. It was also demonstrated that ischemia may be more easily discriminated using 3D-CTA compared with CCA (Fig. 2C). These outcomes indicate that 3D-CTA could efficiently evaluate the risk events for patients with acute CAD.

3D-CTA for the diagnosis of patients with acute CAD. The accuracy of 3D-CTA as a diagnostic tool for patients with acute CAD was investigated. 3D-CTA was used to successfully diagnose 86 patients with acute CAD, 34 patients with myocardial infarction and 16 patients with stable angina. CCA successfully diagnosed 72 patients with acute CAD, 26 with 


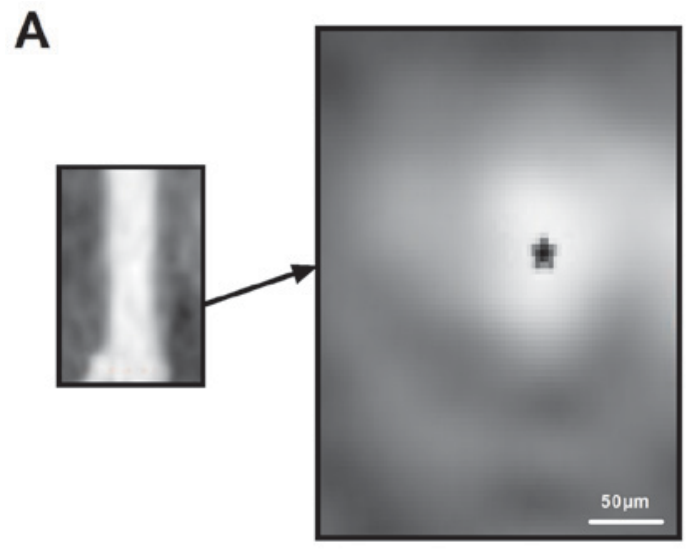

CCA
B

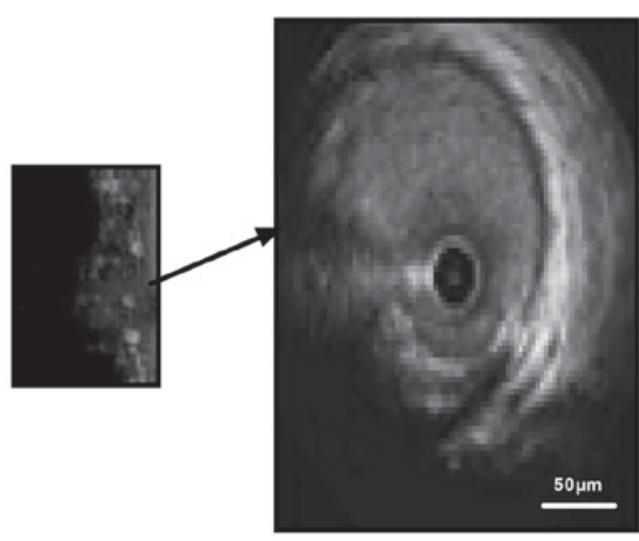

3D-CTA

C

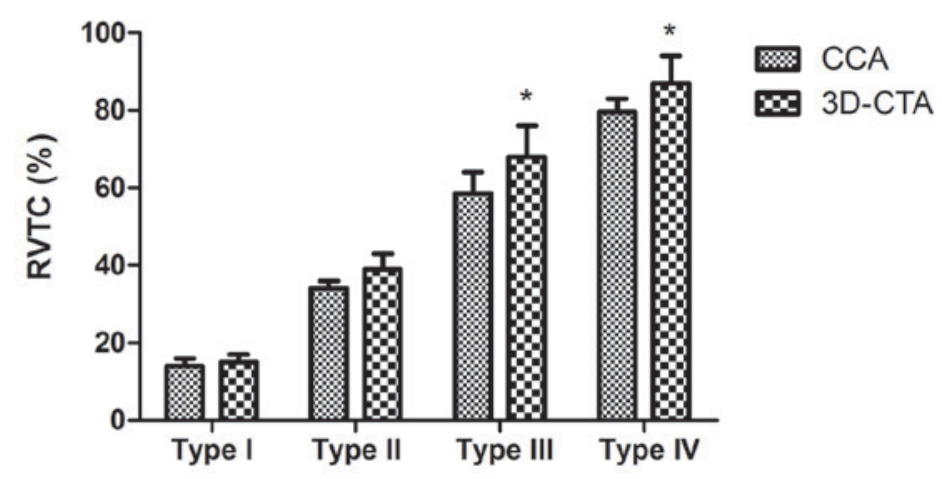

Figure 1. Analysis of calcified plaques in patients with acute coronary artery disease using (A) CCA and (B) 3D-CTA. (C) RVTC in patients with acute coronary artery disease. Magnification, $\mathrm{x} 40$. " $\mathrm{P}<0.05$ vs. CCA. CCA, conventional coronary angiography; 3D-CTA, three-dimensional computed tomography angiography; RVTC, calcified plaque volume to vessel circumference.

A

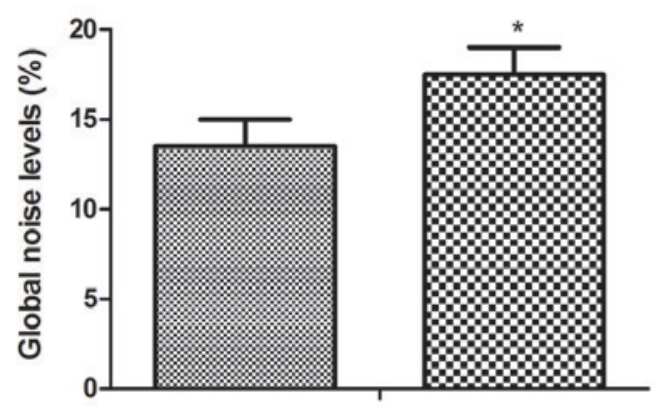

C
B
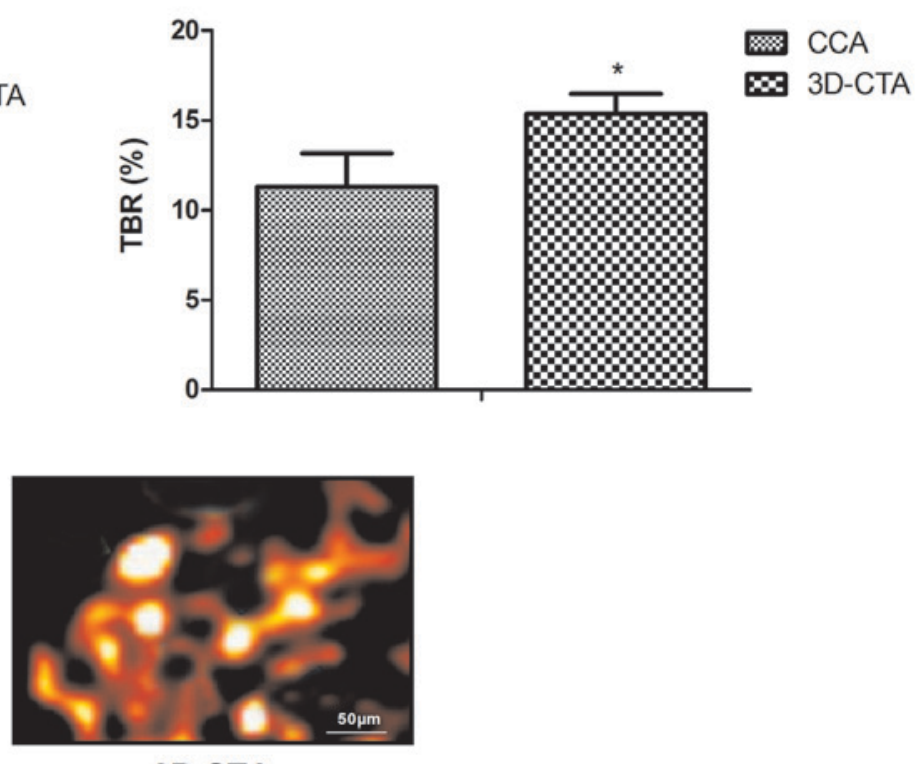

3D-CTA

Figure 2. Efficacy of 3D-CTA for evaluating risk events in patients with acute coronary artery disease. (A) Global noise levels, (B) TBR and (C) discrimination of ischemia in patients with acute coronary artery disease. ${ }^{*} \mathrm{P}<0.05$ vs. CCA. CCA, conventional coronary angiography; $3 \mathrm{D}-\mathrm{CTA}$, three-dimensional computed tomography angiography; TBR, target-to-background ratio.

myocardial infarction and 8 with stable angina (Table III). As such, 3D-CTA had a significantly higher sensitivity for the diagnosis of acute CAD compared with CCA in the present study $(\mathrm{P}<0.01$; Fig. 3$)$. Taken together, these results suggest 
Table III. Diagnostic outcomes between 3D-CTA and CCA for patients with acute coronary artery disease.

\begin{tabular}{lrcc}
\hline Disease & CCA & 3D-CTA & P-value \\
\hline Acute coronary artery disease & 72 & 86 & 0.0372 \\
Myocardial infarction & 26 & 34 & 0.0460 \\
Stable angina & 8 & 16 & 0.0174 \\
\hline
\end{tabular}

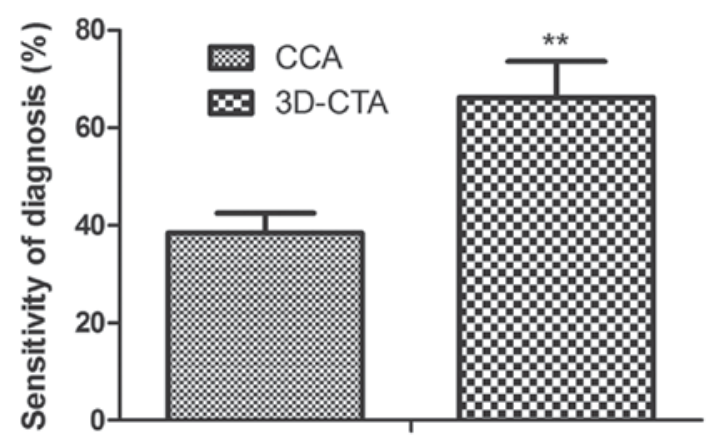

Figure 3. Diagnostic accuracy of 3D-CTA for patients with acute coronary artery disease. ${ }^{* *} \mathrm{P}<0.01$ vs. CCA. CCA, conventional coronary angiography; 3D-CTA, three-dimensional computed tomography angiography.

that 3D-CTA may be used in place of CCA to improve the diagnostic accuracy for patients with acute CAD.

\section{Discussion}

Currently, acute CAD has high mortality and is associated with metabolic disorders of myocardial cells (23). Early diagnosis of acute CAD is important to improve treatment (24). It has been reported that 3D-CTA is a useful tool for aiding doctors in recognizing the morphology of coronary arteries and plaques in patients with CAD (25). In the present study, the diagnostic efficacy of 3D-CTA for acute CAD was assessed, using CCA diagnosis as a control. The results revealed that 3D-CTA had a significantly greater diagnostic accuracy than CCA for patients with acute CAD.

Visualization techniques utilizing multislice CT-coronary angiography of the heart are beneficial for identifying correlations between axial, multiplanar, 3D and virtual endoscopic imaging (26). In the present study, 3D-CTA data was used to diagnose 86 patients with acute $\mathrm{CAD}, 34$ with myocardial infarction and 16 with stable angina with significantly greater accuracy than CCA. 3D-CTA also presented a direct role during the surgery for patients with CAD (20). The results demonstrate that 3D-CTA is able to accurately identify the calcified plaque and RVTC for patients with acute CAD, which may be an efficient method for the diagnosis of acute CAD patients.

Acute coronary syndrome is a kind of cardiovascular disease caused by the rupture of coronary atherosclerosis plaques (27). It has previously been reported that 3D-CTA produces high quality 3D cardiac images that can be used to predict the contrast injection rate for patients with congenital heart disease (28). It the present study it was revealed that the diagnostic accuracy and specificity of 3D-CTA is significantly higher for acute CAD patients compared with CCA. 3D-CTA clearly identified global noise levels, TBR and ischemia in patients with acute CAD. Notably, 3D-CTA had a greater diagnostic sensitivity for acute CAD compared with CCA. However, further research should be conducted to authenticate the diagnostic efficacy of 3D-CTA for patients with acute CAD. Additionally, receiver operator characteristic curves should be plotted to compare the sensitivity and specificity of CCA and 3D-CTA using a larger sample size of patients with suspected acute CAD.

In summary, 3D-CTA may be used to clinically diagnosis patients with acute CAD. 3D-CTA may improve the overall diagnostic accuracy and contribute to treatment for acute patients with CAD, However, further studies should be performed to confirm this.

\section{Acknowledgements}

Not applicable.

Funding

No funding was received.

\section{Availability of data and materials}

The datasets used and/or analyzed during the current study are available from the corresponding author on reasonable request.

\section{Authors' contributions}

LL performed the experiments. JH and SQ analyzed data. YF designed the study.

\section{Ethics approval and consent to participate}

The Ethical Committee of the Affiliated Hospital of Changchun University of Chinese Medicine approved this prospective study. Written informed consent was obtained from all the patients.

\section{Consent for publication}

Written informed consent was obtained from all the patients.

\section{Competing interests}

The authors declare that they have no competing interests.

\section{References}

1. Dickens C, Cherrington A and McGowan L: Depression and health-related quality of life in people with coronary heart disease: A systematic review. Eur J Cardiovasc Nurs 11: 265-275, 2012.

2. Zwadlo C, Meyer GP, Schieffer B and Westhoff-Bleck M: Anomalous intramural course of coronary arteries in congenital heart disease-three case reports and review of the literature. Congenit Heart Dis 7: 139-144, 2012.

3. Mehra M, Somohano T and Choi M: Mandibular fibular graft reconstruction with CAD/CAM technology: A clinical report and literature review. J Prosthet Dent 115: 123-128, 2016. 
4. Haasenritter J, Aerts M, Bösner S, Buntinx F, Burnand B, Herzig L, Knottnerus JA, Minalu G, Nilsson S, Renier W, et al: Coronary heart disease in primary care: Accuracy of medical history and physical findings in patients with chest pain-a study protocol for a systematic review with individual patient data. BMC Fam Pract 13: 81, 2012.

5. Fan J, Song Y, Wang Y, Hui R and Zhang W: Dietary glycemic index, glycemic load, and risk of coronary heart disease, stroke, and stroke mortality: A systematic review with meta-analysis. PLoS one 7: e52182, 2012.

6. Ciatto S, Rosselli Del Turco M, Burke P, Visioli C, Paci E and Zappa M: Comparison of standard and double reading and computer-aided detection (CAD) of interval cancers at prior negative screening mammograms: Blind review. Br J Cancer 89 $1645-1649,2003$

7. Janssen V, De Gucht V, Dusseldorp E and Maes S: Lifestyle modification programmes for patients with coronary heart disease: A systematic review and meta-analysis of randomized controlled trials. Eur J Prev Cardiol 20: 620-640, 2013

8. Maddahi J and Packard RR: PET should replace SPECT in cardiac imaging for diagnosis and risk assessment of patients with known or suspected CAD: Pro. J Nucl Cardiol 24: 1955-1959, 2017.

9. Schelbert H: Measurement of MBF by PET is ready for prime time as an integral part of clinical reports in diagnosis and risk assessment of patients with known or suspected CAD: For prime time not yet: Need impact and certainty. J Nucl Cardiol 25 $153-156,2018$

10. Davari Dolatabadi A, Khadem SEZ and Asl BM: Automated diagnosis of coronary artery disease (CAD) patients using optimized SVM. Comput Methods Programs Biomed 138: 117-126, 2017.

11. Ni J and Wang P: Reasonable application of cardiac CT in the diagnosis of coronary atherosclerotic heart disease. Zhonghua $\mathrm{Yi}$ Xue Za Zhi 95: 801-802, 2015 (In Chinese).

12. Perkins JA, Sidhu M, Manning SC, Ghioni V and Sze R: Three-dimensional CT angiography imaging of vascular tumors of the head and neck. Int J Pediatr Otorhinolaryngol 69: 319-325, 2005.

13. Murai S, Hamada S, Yamamoto S, Khankan AA, Sumikawa H, Inoue $\mathrm{A}$, Tsubamoto $\mathrm{M}$, Honda $\mathrm{O}$, Tomiyama $\mathrm{N}$, Johkoh $\mathrm{T}$ and Nakamura H: Evaluation of major aortopulmonary collateral arteries (MAPCAs) using three-dimensional CT angiography: Two case reports. Radiat Med 22: 186-189, 2004.

14. Kato Y, Hayakawa M and Katada K: Three-dimensional multislice helical CT angiography of cerebral aneurysms. Nihon Rinsho 62: 715-721, 2004 (In Japanese).

15. Jeong Y, Lim C, Oh S, Jung J, Chang J, Yoon J and Choi M: Three-dimensional CT angiography of the canine hepatic vasculature. J Vet Sci 9: 407-413, 2008.

16. Rozen WM, Stella DL, Ashton MW, Phillips TJ and Taylor GI Three-dimensional CT angiography: A new technique for imaging microvascular anatomy. Clin Anat 20: 1001-1003, 2007.

17. Fishman EK, Horton KM and Johnson PT: Multidetector CT and three-dimensional CT angiography for suspected vascular trauma of the extremities. Radiographics 28: 653-665, 2008.
18. Tanabe S, Uede T, Nonaka T, Ohtaki M and Hashi K: Diagnosis of cerebral arteriovenous malformations with three-dimensional CT angiography. J Clin Neurosci 5 (Suppl): S33-S38, 1998

19. Prestigiacomo CJ, Sabit A, He W, Jethwa P, Gandhi C and Russin J: Three dimensional CT angiography versus digital subtraction angiography in the detection of intracranial aneurysms in subarachnoid hemorrhage. J Neurointerv Surg 2: 385-389, 2010.

20. Mari FS, Nigri G, Pancaldi A, De Cecco CN, Gasparrini M, Dall'Oglio A, Pindozzi F, Laghi A and Brescia A: Role of CT angiography with three-dimensional reconstruction of mesenteric vessels in laparoscopic colorectal resections: A randomized controlled trial. Surg Endosc 27: 2058-2067, 2013.

21. Hoffmann MH, Shi H, Schmid FT, Gelman H, Brambs HJ and Aschoff AJ: Noninvasive coronary imaging with MDCT in comparison to invasive conventional coronary angiography: A fast-developing technology. AJR Am J Roentgenol 182: 601-608, 2004.

22. Matsumoto M, Kodama N, Sakuma J, Sato S, Oinuma M, Konno Y, Suzuki K, Sasaki T, Suzuki K, Katakura T and Shishido F: 3D-CT arteriography and 3D-CT venography: The separate demonstration of arterial-phase and venous-phase on 3D-CT angiography in a single procedure. AJNR. Am J Neuroradiol 26: 635-641, 2005.

23. Wang Z, Zhang J, Ren T and Dong Z: Targeted metabolomic profiling of cardioprotective effect of Ginkgo biloba L. extract on myocardial ischemia in rats. Phytomedicine 23: 621-631, 2016.

24. Hashoul S, Gaspar T, Halon DA, Lewis BS, Shenkar Y, Jaffe R, Peled N and Rubinshtein R: Automated computer-assisted diagnosis of obstructive coronary artery disease in emergency department patients undergoing 256-slice coronary computed tomography angiography for acute chest pain. Am J Cardiol 116: 1017-1021, 2015.

25. Athanasiou LS, Rigas GA, Sakellarios AI, Exarchos TP, Siogkas PK, Michalis LK, Parodi O, Vozzi F and Fotiadis DI: Three-dimensional reconstruction of coronary arteries and plaque morphology using CT angiography-comparison and registration using IVUS. Conf Proc IEEE Eng Med Biol Soc 2015: 5638-5641, 2015

26. Herzog C, Ay M, Engelmann K, Abolmaali N, Dogani S, Diebold T and Vogl TJ: Visualization techniques in multislice CT-coronary angiography of the heart. Correlations of axial, multiplanar, three-dimensional and virtual endoscopic imaging with the invasive diagnosis. Rofo 173: 341-349, 2001 (Article in German)

27. Thompson KA, Philip KJ, Barbagelata A and Schwarz ER: Review article: The new concept of interventional heart failure therapy-part 1: Electrical therapy, treatment of CAD, fluid removal, and ventricular support. J Cardiovasc Pharmacol Ther 15: 102-111, 2010.

28. Jelnin V, Co J, Muneer B, Swaminathan B, Toska S and Ruiz CE: Three dimensional CT angiography for patients with congenital heart disease: Scanning protocol for pediatric patients. Catheter Cardiovasc Interv 67: 120-126, 2006. 